\title{
NOTES
}

\section{Upper Critical Solution Temperature Behavior in Polystyrene/Poly(methyl methacrylate) Mixture}

\author{
Toshiaki OugrzaWA ${ }^{\dagger}$ and David J. WALSH* \\ Polymer Processing Laboratory, National Institute of Materials and Chemical Research, \\ Tsukuba, Ibaraki 305, Japan \\ * Du Pont Polymers, Sabine Research Laboratory, \\ PO Box 1089, Orange, Texas 77631-1089, U.S.A.
}

(Received April 2, 1993)

\begin{abstract}
KEY WORDS Phase Diagram / Cloud-Point Curve / Upper Critical Solution Temperature / Polystyrene / Poly(methyl methacrylate) / Oligomer Mixture / Equation of State Theory / Interaction Parameter /
\end{abstract}

It is well-known that most pairs of high molar mass polymers are immiscible. This is so because the combinatorial entropy of mixing of two polymers is dramatically less than that for two low molar mass compounds. ${ }^{1}$ The enthalpy of mixing, on the other hand, is often a positive quantity. Therefore dissimilar polymers are only miscible if there are favorable specific interactions between them leading to a negative contribution to the Gibbs free energy of mixing. Miscible polymers tend to phase separate at elevated temperatures. This lower critical solution temperature (LCST) behavior is typical for miscible polymer blends. The LCST behavior is interpreted in terms of the equation of state or free volume contribution. ${ }^{2,3}$ Some miscible polymers also exhibit phase separation on cooling temperatures. This upper critical solution temperature (UCST) behavior has been observed even for the polymer mixtures with high molar mass, but generally when one or two components are low molar mass, i.e., oligomers. ${ }^{4,5}$ A typical example of UCST behavior was obtained for polystyrene(PS)/polybutadiene(PB) mixtures, ${ }^{4}$ but the mixtures for high molar mass

\footnotetext{
† To whom correspondence should be addressed.
}

are immiscible except for the extreme ends of composition. This behavior can be interpreted by both the Flory-Huggins expression and the equation of state theories.

It is also well-known that PS and poly(methyl methacrylate) (PMMA) are immiscible but the interaction parameter between these polymers is not so large. It has been reported that, at ambient temperature, lower molecular weight PS samples could be miscible with the PMMA samples within limited composition ranges. ${ }^{6}$ However, an actual UCST type phase diagram has not been reported yet. The measurements of the Flory-Huggins interaction parameter $\chi$ were also carried out by using a number of different methods, for example, the measurements of interfacial thickness in the two phase state, ${ }^{7}$ light scattering on ternary polymer solutions including solvent ${ }^{8}$ and neutron scattering from the homogeneous state in a block copolymer of styrene and methyl methacrylate. ${ }^{9}$ The values of the $\chi$, themselves not so large, exhibit some scatter depending on the techniques, but are positive and decrease with increasing temperature. One can predict that the UCST phase diagram exists for an 
Table I. Specifications of materials and their equation of state parameters

\begin{tabular}{llrccccc}
\hline Polymer & Source & $M_{w}$ & $M_{w} / M_{n}$ & $P^{*} / \mathrm{MPa}$ & $V_{\mathrm{sp}}{ }^{*} / \mathrm{cm}^{3} \mathrm{~g}^{-1}$ & $T^{*} / \mathrm{K}$ & $S_{\mathrm{S}} / S_{\mathrm{MMA}}$ \\
\hline PS0.8k & Aldrich & 794 & 1.13 & 470 & 0.8457 & 7212 & $0.83^{\mathrm{a}}$ \\
PMMA6k & Polysciences & 6350 & 1.06 & $600^{\mathrm{b}}$ & $0.7224^{\mathrm{b}}$ & $7748^{\mathrm{b}}$ & - \\
PMMA12k & Polysciences & 12900 & 1.04 & 600 & 0.7224 & 7748 & - \\
\hline
\end{tabular}

a Calculated by Bondi's group contribution data. ${ }^{17}$

b Since the values of characteristic parameters are almost constant for PMMA which has a molecular weight more than about five thousand, we used the same values as those of PMMA12k for PMMA6k.

oligomer mixture of PS/PMMA as well as for a PS/PB mixture.

Here we describe results of UCST behavior for a PS/PMMA oligomer mixture. To our knowledge, this is the first observation of a UCST phase diagram in a PS/PMMA mixture. On the basis of the phase diagrams, we simulate spinodal and binodal curves for this system by using the equation of state theory and estimate the value of the interaction parameter $X_{12}$ (not the $\chi$ parameter).

The characteristics of polymer samples used in this study are shown in Table I. PS and PMMA were dissolved at $5 \mathrm{wt} \%$ of total polymer in tetrahydrofuran. The solution was cast onto a cover glass. A quick evaporation of solvent makes the sample film transparent but a slow one makes it opaque. The light scattering profile of the opaque film by a goniometer trace often exhibits a peak; furthermore the modulated structure was observed in the optical microscope, as in the $\mathrm{PB} /$ poly(styrene-co-butadiene) system by casting from toluene solution. ${ }^{10}$ This implies that the phase separation takes place by the spinodal decomposition during casting. The cast film was further dried under vacuum. Then the film on the cover glass was inserted in a heating stage setting horizontally on the light scattering stage. ${ }^{10} \mathrm{~A} \mathrm{He}-\mathrm{Ne}$ gas laser beam of $632.8 \mathrm{~nm}$ wavelength was applied vertically to the film specimen. The phase separated opaque samples were heated with a constant rate of $1 \mathrm{~K} \mathrm{~min}^{-1}$ and the temperature at which phase dissolution occurs was recorded by a decrease

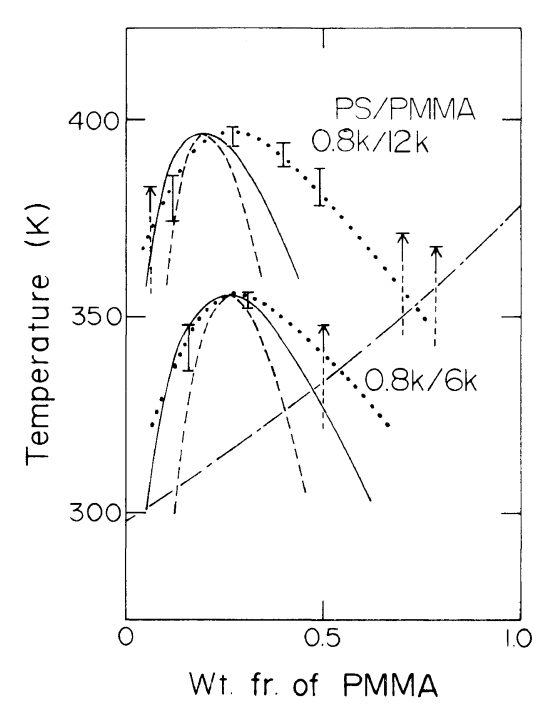

Figure 1. Cloud point curves (dotted) and simulated binodal (-) and spinodal (------) curves of PS/PMMA mixtures. Experimental cloud point data are shown by solid bars and arrows indicate data obtained from only heating measurement. $T_{\mathrm{g}}$ for one phase mixture calculated by Fox equation ${ }^{12}$ (_- $)$ are also shown.

in the intensity of the scattered light from samples. ${ }^{11}$ Next the transparent samples at high temperature were cooled with a constant rate of $1 \mathrm{~K} \mathrm{~min}^{-1}$ and the temperature at which phase separation occurs was recorded by an increase in the intensity of the scattered light. These temperatures correspond to cloud points and are indicated as solid bars in Figure 1. The upper end of the bar refers to phase dissolution temperature obtained on heating, and the lower end to phase separation temperature obtained on cooling.We also checked the reversibility of the transparent-opaque transition correspond- 
ing to the one-two phase in every case except for the composition in both the vicinity of the glass transition temperature $\left(T_{\mathrm{g}}\right)$ of the blends and the extreme end of the phase diagram. The difference between the cloud-point temperatures for heating and cooling measurements is large in end compositions of phase diagram. In Figure 1, the cloud point curves corresponding to the binodal curve are drawn somewhat arbitrarily (dotted curves). Thus we have found a UCST phase diagram for PS/PMMA systems.

We simulate the binodal and spinodal curves in the phase diagram of PS/PMMA mixtures by using the equation of state theory of Flory, Orwoll and Vrij (FOV). ${ }^{13}$ Since the detail of the formulae were described in previous papers, ${ }^{14,15}$ we indicate only the main equations. The FOV equation of state is given by

$$
\tilde{P} \tilde{V} / \tilde{T}=\tilde{V}^{1 / 3}\left(\tilde{V}^{1 / 3}-1\right)-1 /(\tilde{T} \tilde{V})
$$

where $\tilde{P}=P / P^{*}, \check{V}=v / v^{*}$, and $\tilde{T}=T / T^{*}$ are the reduced pressure, volume and temperature, $P$, $v$ and $T$ are the actual values, and $P^{*}, v^{*}$, and $T^{*}$ are the hard-core values (characteristic parameters). When calculating the free energy change on mixing of two substances to produce a homogeneous blend, one assumes that the mixture obeys the same equation of state as the pure components, that the hard-core volumes are additive, and that the interatomic interactions can be assumed in such a way that hard-core pressure of the mixture is given by

$$
P^{*}=\phi_{1} P_{1}{ }^{*}+\phi_{2} P_{2}{ }^{*}-\phi_{1} \theta_{2} X_{12}
$$

where $\phi_{i}$ and $\theta_{i}$ is a hard-core volume fraction and a site fraction of component $i$, respectively, and $X_{12}$ is an interaction parameter and different from the Flory-Huggins $\chi$ parameter. The resulting expression for the chemical potential of the mixture is

$$
\begin{aligned}
& \Delta \mu_{1} / R T=\ln \phi_{1}+\left(1-r_{1} / r_{2}\right) \phi_{2} \\
& \quad+P_{1} * V_{1} * / R T\left\{3 \tilde{T}_{1} \ln \left[\left(\tilde{V}_{1}^{1 / 3}-1\right) /\left(\tilde{V}^{1 / 3}-1\right)\right]\right. \\
& \quad+1 / \tilde{V}_{1}-1 / \tilde{V}+\tilde{P}_{1}\left(\tilde{V}-\tilde{V}_{1}\right) \\
& \left.\quad+\phi_{2} \theta_{2} X_{12} /\left(P_{1} * \tilde{V}\right)\right\}
\end{aligned}
$$

Table II. Values of interaction parameter

\begin{tabular}{cc}
\hline Mixture & $X_{12} / \mathrm{J} \mathrm{cm}^{-3}$ \\
\hline PS0.8k/PPMA6k & 3.77 \\
PS0.8k/PPMA12k & 3.45 \\
\hline
\end{tabular}

where $r_{i}$ is the number of segments per molecule. The first two terms in eq 3 represent the contribution of the combinatorial entropy, the last term containing $X_{12}$ represents the contribution of the interactions to the chemical potential change, and the other terms represent the contribution of free volume.

From eq 3 one can calculate the phase diagram for binary systems. To do this, the characteristic parameters of pure components and $X_{12}$ are necessary. The characteristic parameters of pure components can be obtained from the fitting pressure-volumetemperature (PVT) data to the FOV equation of state as described in a previous paper. ${ }^{16}$ The value of $X_{12}$ for PS/PMMA system has not been known. On the other hand, we can estimate the values of $X_{12}$ required to locate the phase diagaram in the correct temperature range from simulation by using eq 3 .

The values of the parameters used in this simulation are shown in Table I. $S_{i} / S_{j}$ is the ratio of the surface area per unit volume and used in order to obtain the value of $\theta_{i}$. Calculated binodal and spinodal curves are shown in Figure 1 and the values of $X_{12}$ used to simulate the phase diagrams are shown in Table II. These values of $X_{12}$ are small in comparison with those of $\mathrm{PS} / \mathrm{PB}\left(X_{12}=7.0\right.$ $\mathrm{J} \mathrm{cm}^{-3}$ ) and methoxylated poly(ethylene glycol)/poly (propylene glycol) $\left(X_{12}=10-13\right.$ $\left.\mathrm{J} \mathrm{cm}^{-3}\right),{ }^{18}$ as predicted. The shapes of the simulated phase diagrams are not identical with that of the measured cloud point curves but the compositions at the maximum of the phase diagrams are close in both mixtures. The fit for PS0.8k/PMMA6k is good considering the composition in the vicinity of $T_{\mathrm{g}}$. Since the difference between the values of $T^{*}$ in PS and 
PMMA is small, the contribution of the free volume terms in eq 3 is small, especially for high molecular weight polymer mixtures. Therefore the contribution of the $X_{12}$ term (interaction) is dominant for miscibility in this mixture though the value of $X_{12}$ is not so large. Considering the contribution of a positive $X_{12}$ to the $\chi$ parameter, ${ }^{19}$ that could be in agreement with the result of Russell et al. that the value of $\chi$ decreases with increasing temperature in a PS/PMMA mixture. ${ }^{9}$

\section{REFERENCES}

1. P. J. Flory, "Principles of Polymer Chemistry," Cornell University Press, Ithaca, New York, N.Y., 1953.

2. D. R. Paul and S. Newman, Ed. "Polymer Blends," Vol. 1, Academic Press, New York, N.Y., 1978; Vol. 1.

3. O. Olabisi, L. M. Robeson, and M. T. Shaw, "Polymer-Polymer Miscibility," Academic Press, New York, 1979.

4. R. J. Roe and W. C. Zin, Macromolecules, 13, 1221 (1980).

5. S. L. Zacharius, G. ten Brinke, W. J. MacKnight, and F. E. Karasz, Macromolecules, 16, 381 (1983).

6. E. V. Thompson, "Polymer Alloys," Vol. II, D. Klempner and K. C. Frisch, Ed., Plenum Press, New York, 1979, p 1.

7. N. Higashida, J. Kressler, S. Yukioka, and T. Inoue, Macromolecules, 25, 5259 (1992).

8. T. Fukuda, M. Nagata, and H. Inagaki, Macromolecules, 17, 548 (1984).

9. T. P. Russell, R. P. Hjelm, and P. A. Seeger, Macromolecules, 23, 890 (1990).

10. T. Inoue, T. Ougizawa, O. Yasuda, and K. Miyasaka, Macromolecules, 18, 57 (1985).

11. T. Ougizawa, T. Inoue, and H. W. Kammer, Macromolecules, 18, 2089 (1985).

12. T. G. Fox, Bull. Am. Phys. Soc., 1, 123 (1956).

13. P. J. Flory, R. A. Orwoll, and A. Vrij, J. Am. Chem. Soc., 86, 3507 and 3515 (1964).

14. D. J. Walsh, G. T. Dee, J. L. Halary, J. M. Ubrich, M. Millequant, J. Lesec, and L. Monnerie, Macromolecules, 22, 3395 (1989).

15. D. J. Walsh, G. T. Dee, and T. Ougizawa, Makromol. Chem., Macromol. Symp., 38, 255 (1990).

16. G. T. Dee and D. J. Walsh, Macromolecules, 21, 811 (1988).

17. A. Bondi, J. Phys. Chem., 68, 441 (1964).

18. S. Rostami and D. J. Walsh, Macromolecules, 18, 1228 (1985).

19. D. Patterson and A. Robard, Macromolecules, 11, 690 (1978). 